\title{
Residual Energy-Aware Cooperative Transmission (REACT) in Wireless Networks
}

\author{
Erwu Liu ${ }^{\dagger}$, Qinqing Zhang ${ }^{\ddagger}$ and Kin K. Leung ${ }^{\dagger}$ \\ ${ }^{\dagger}$ Department of Electrical and Electronic Engineering, Imperial College, London, UK \\ Email:\{erwu.liu, kin.leung\}@imperial.ac.uk \\ ${ }^{\ddagger}$ Applied Physics Laboratory, Johns Hopkins University, USA \\ ${ }^{\ddagger}$ Department of Computer Science, Johns Hopkins University, USA \\ Email:qinqing.zhang@jhuapl.edu
}

\begin{abstract}
We study the problem of cooperative transmission, relay selection and scheduling in battery-operated wireless networks, where energy efficiency is a critical design consideration. We assume that multiple relay nodes in the network can cooperate their transmissions of information to gain energy savings in a distributed network MIMO system. We formulate the problem as maximizing the lifetime of the network in terms of maximizing the overall number of packets transmitted by the source to the destination, given a limited energy supply at the source node and each relay node along the transmission path. Unlike the existing method where all relay nodes cooperatively transmit to the receiving node, we construct a selection method to choose a subset of all relays to participate in the cooperative transmission. The chosen subset is the one that maximizes the lifetime of the network and we call the selection method a residual energy-aware cooperative transmission (REACT) algorithm. Our simulation results show that the proposed REACT algorithm demonstrate much improved lifetime compared to the existing method.
\end{abstract}

\section{INTRODUCTION}

Energy conservation is crucial in extending the lifetime of battery-operated wireless networks. To achieve high energy efficiency, approaches have been proposed to tackle this problem from different angles across the layers from hardware up to the application. These include efficient energy management techniques for peripheral devices on handheld and embedded hardware platforms [1], energy efficient medium access control design [2], routing algorithm [3], and energy efficient algorithms for broadcast and multicast applications [4]. While each of these areas has received a lot of attention separately in recent years, a joint design of energy efficiency cross the layers remains very limited due to the complexity of this problem. Our aim in this paper is to tackle the energy efficiency problem from cooperative transmission, relay selection, and scheduling perspectives in a battery-powered wireless network.

Cooperative diversity [5], [6], [7] is a new form of diversity through distributed transmission and processing with node collaboration. Transmit cooperation has nodes exchanging each other's messages, sharing their antennas, and creating multiple paths to transmit the information. Receive cooperation has nodes forwarding information about their observations for

This work was supported, in part, by Johns Hopkins University, Applied Physics Laboratory's internal research and development funds. decoding. A system with both transmit and receive cooperation is similar to a multiple-input multiple-output (MIMO) system in a networked manner. Therefore it is sometimes called a distributed MIMO or network MIMO. It is known that transmit and receiver diversity can achieve higher capacity without sacrificing bandwidth or energy. In a network where each node is equipped with a single omnidirectional antenna, cooperative diversity can achieve similar gains from a MIMO system where each node is equipped with multiple antennas. However, there are several limitations that hurdle the potential gains in a network MIMO compared to the conventional MIMO system, due to the absence of a direct high capacity connection (e.g., usually a cable) among the antenna elements. For example, synchronization among the distributed antenna is much harder than a conventional MIMO transmitter. Additional resources such as bandwidth, power and time are required to enable the cooperation. Antenna power allocation cannot be done as that in a conventional MIMO system, etc. In this paper, we shall focus on the energy savings from cooperative diversity in an optimal way. Similar to [8], we do not consider the effects of those limitations and assume that an appropriate architecture for achieving the required level of coordination among the cooperative nodes can take place [9].

Khandani, et al [8] proposed a design of cooperative diversity to maximize energy savings. They formulated the problem as minimizing the overall energy consumption of the relay nodes between a transmission node and a receiving node. In this formulation, all relay nodes participate in cooperative transmission to gain energy savings. Further, relay nodes with very good channel conditions tend to participate more (e.g., transmit more often) than other nodes with poor channel conditions, since the good nodes are expected to use less transmission power than the poor nodes. This method achieves the overall savings of energy consumed by all the participating nodes. However, it may not be suitable for battery-powered nodes in an ad hoc or sensor network, where each node has its own limit of energy conservation. In fact, since nodes with good channel conditions transmit more often than others, these nodes will run out of their battery power more quickly. As a result, the number of cooperative relay nodes will decrease and cause an increase of the aggregate transmission power, 
which then again cause the remaining good nodes to run out of battery power quickly.

Our goal in this paper is to address the similar energy saving problem with consideration of each node's limited power conservation. We formulate the problem of energy savings as maximizing the lifetime of the network in terms of maximizing the overall number of packets transmitted by the source node to the destination, given a limited energy supply for the source node and each relay node along the optimal transmission path. To solve this problem, an intuitive solution is that we only select a subset of relay nodes to participate in the cooperative transmission, and thus avoid the overuse of the good nodes in the set at each transmission stage. Unlike selective relaying in [7] where a relay with highest capacity is selected, we construct a selection algorithm of the subset of nodes based on the residual energy of the nodes at each transmission stage. In our method, we trade a small portion of cooperative diversity gain with a much improved energy saving at each individual node, while still taking the wireless boardcast advantage (WBA) [4] and wireless cooperative advantage (WCA) [8]. Note that our method may not achieve the optimal energy savings in terms of total power consumptions by all participating nodes as in method [8]. However, our method will achieve much improved lifetime in terms of preserving the residual energy of each node. Based on this consideration, we call it a residual energyaware cooperative transmission (REACT) algorithm.

The rest of the paper is structured as follows. In Section II, we first introduce basic terminology and concepts of cooperative transmission in relay-aided wireless networks, we then show that the traditional method is not suitable for battery-powered wireless networks. After that, residual energyaware cooperative transmission (REACT) is proposed to avoid overly-used relays while it still benefits from the traditional cooperative transmission techniques. Finally, in Section III, we present simulation results to illustrate that the REACT algorithm is energy-efficient for both Rayleigh fading and nonfading environment, followed by the conclusion in Section IV.

\section{SySTEM MODEL}

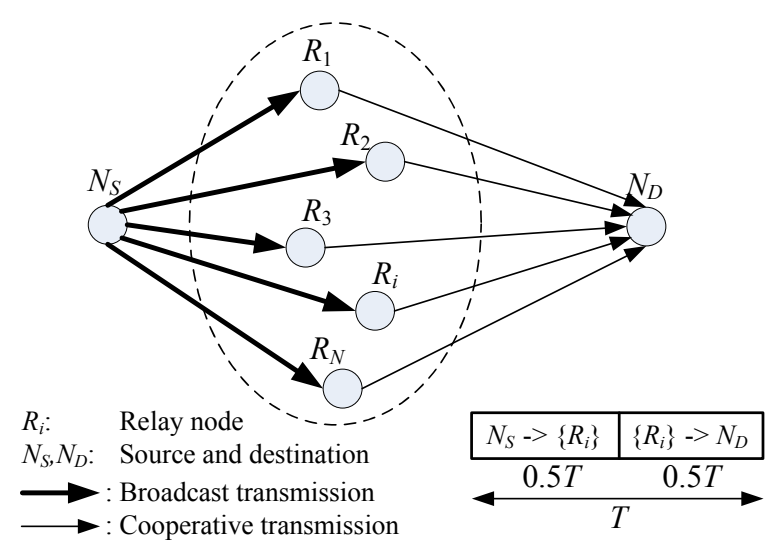

Fig. 1. Cooperative Transmission in Wireless Networks

Refer to Fig. 1 for a two-hop cooperative transmission.
Without relays $R_{1} \sim R_{N}$, Nodes $N_{S}$ and $N_{D}$ are the 1hop neighbors in an ad hoc (or sensor) network. Consider the problem that Node $N_{S}$ wants to transmit packets to Node $N_{D}$, with the help of $N$ relays $R_{i}(i=1,2, \ldots, N)$. Since this two-hop cooperative transmission can be easily extended to the multi-hop case [8], we in this paper focus on two-hop cooperative transmission. As shown in Fig. 1, each transmission slot $T$ is equally divided into two parts. At the first half slot, $N_{S}$ broadcasts signals with power $P_{B L}$ to relay nodes denoted as $R_{1}, R_{2}, \ldots, R_{N}$. At the second half slot, relay nodes which have successfully received \& decoded the packets sent at the first half slot will cooperatively transmit to $N_{D}$. For a relay to successfully receive $\&$ decode signals over broadcast link, we assume that the received signal-to-noiseratio $(S N R)$ must be at least $S N R_{\min }$. i.e.,

$$
\frac{P_{B L} \times\left|h_{S, i}\right|^{2}}{N_{0} \times W} \geq S N R_{\text {min }}, \forall i, 1 \leq i \leq N .
$$

where $h_{S, i}$ is the channel gain of the link $N_{S} \rightarrow R_{i}, N_{0}$ is the noise power density and $W$ is the bandwidth.

Similarly, for a destination node to successfully receive \& decode signals over cooperative link, the received $S N R$ must be at least $S N R_{\min }$. In [8], Khandani assumes in-phase receiving, i.e., signal amplitudes are added at the destination node. We use the same assumption and have,

$$
\frac{\left(\sum_{i=1}^{N}\left(\sqrt{P_{i}} \times\left|h_{i, D}\right|\right)\right)^{2}}{N_{0} \times W} \geq S N R_{m i n}, \forall i, 1 \leq i \leq N .
$$

where $P_{i}$ is the transmit power of $R_{i}, h_{i, D}$ is the channel gain of link $R_{i} \rightarrow N_{D}$.

Given the above constrains, with lagrangian multiplier techniques, it is easy to prove that the aggregate transmit power of all relays is minimized if

$$
P_{i}=\frac{\left|h_{i, D}\right|^{2}}{S N R_{\min } \times N_{0} \times W} /\left(\sum_{i=1}^{N} \frac{\left|h_{i, D}\right|^{2}}{S N R_{\min } \times N_{0} \times W}\right)^{2} .
$$

In [8], the link cost of a wireless link is defined as the minimum power required to successfully transmit signals over the link. Using the same formulation, we have

$$
\begin{gathered}
L C_{S, i}=\frac{S N R_{\min } \times N_{0} \times W}{\left|h_{S, i}\right|^{2}}, \forall i, 1 \leq i \leq N . \\
L C_{i, D}=\frac{S N R_{\min } \times N_{0} \times W}{\left|h_{i, D}\right|^{2}}, \forall i, 1 \leq i \leq N . \\
L C_{B L}=\max _{1 \leq i \leq N} L C_{S, i} .
\end{gathered}
$$

where $L C_{S, i}, L C_{i, D}$ are the link cost of links $N_{S} \rightarrow R_{i}, R_{i} \rightarrow$ $N_{D}$ and $L C_{B L}$ is the link cost of broadcast link $N_{S} \rightarrow\left\{R_{i}\right\}$.

(3) can be re-written as

$$
P_{i}=\left(\frac{1}{L C_{i, D}}\right) /\left(\sum_{i=1}^{N} \frac{1}{L C_{i, D}}\right)^{2} .
$$




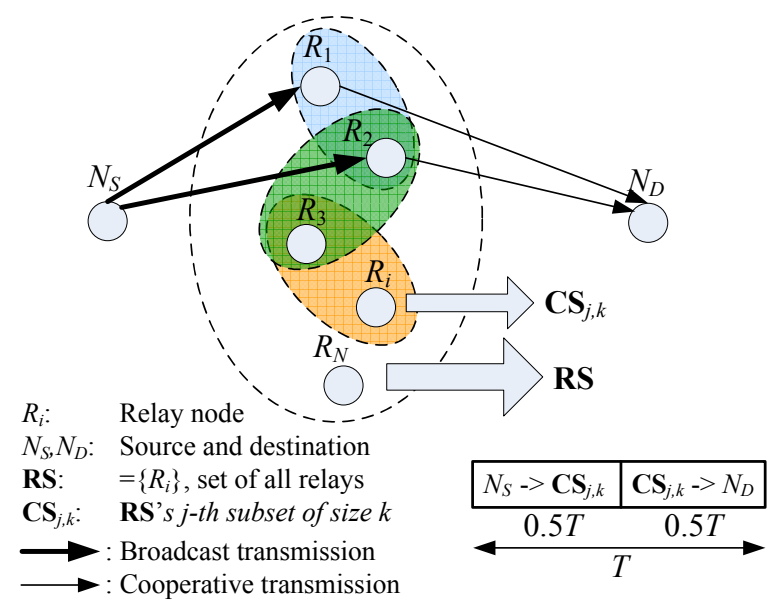

Fig. 2. Residual Energy-Aware Cooperative Transmission (REACT)

Denoted by $L C_{C L}$, the link cost of the cooperative link is

$$
L C_{C L}=\sum_{i=1}^{N} P_{i}=1 /\left(\sum_{i=1}^{N} \frac{1}{L C_{i, D}}\right) .
$$

We assume that the packet size is a bits. Denote $\eta_{S}, \eta_{i}$ to be the required energy for $N_{S}$ and $R_{i}$ to transmit a packet, respectively. Obviously we have

$$
\begin{gathered}
\eta_{S}=L C_{B L} \times \frac{a}{W \times \log _{2}\left(1+S N R_{\text {min }}\right)} . \\
\eta_{i}=P_{i} \times \frac{a}{W \times \log _{2}\left(1+S N R_{\text {min }}\right)} .
\end{gathered}
$$

In [8], all relays participate in cooperative transmission and Relays $R_{i}$ transmit with power $P_{i}$ given by (3). This scheme is not suitable for battery-powered ad-hoc or sensor networks. According to (10), good-channel-condition relays (i.e., those with higher $\left|h_{i, D}\right|^{2}$ ) use more power in cooperative transmission and these relays will run out of their energy and die before bad-channel-condition ones with this scheme. When this happens, the number of cooperative relays will decrease and the aggregate power for cooperative transmission will increase, which makes the remaining relays die quickly. Eventually, $N_{S}$ has to directly transmit to $N_{D}$ as no relay is available and its lifetime could be greatly shorten in this scenario.

An intuitive solution to the above issue is to have those relays with relatively low residual energy not participate in cooperative transmission, while at the same time it should take the wireless broadcast advantage (WBA) [4] and wireless cooperative advantage (WCA) [8]. Based on this, we propose our residual energy-aware cooperative transmission (REACT) algorithm.

As shown in Fig. 2, there are $N$ relays $R_{1}, R_{2}, \ldots, R_{N}$, located between $N_{S}$ and $N_{D}$. We use $\mathbf{R S}=\left\{R_{i}, 1 \leq i \leq M\right\}$ to denote the relay set of all active relays, where $M=|\mathbf{R S}|$ is the total number of relays in RS. Obviously we have $M=N$ initially. Unlike [8] which uses all active relays for cooperative transmission, the REACT algorithm chooses a subset of size $k(1 \leq k \leq M), \mathbf{C S}_{j, k} \subseteq \mathbf{R S}$ to participate in cooperative transmission at a time. Apparently, the REACT algorithm is simply the purely opportunistic transmission method (i.e., no cooperative transmission) for $k=1$, and is simply the traditional cooperative transmission method (i.e., all relays participate in the cooperative transmission) for $k=M$.

When $\mathbf{C S}_{j, k}$ is chosen at slot $t$, Khandani's method [8] is used for the transmission, i.e., $N_{S}$ broadcasts to all relays in $\mathbf{C S}_{j, k}$ at the first half slot of $t$, then all relays in $\mathbf{C} \mathbf{S}_{j, k}$ cooperatively transmit to $N_{D}$ at the second half slot of $t$. For the cooperative subset $\mathbf{C S}_{j, k}$, we use $L C_{B L, j}, L C_{j, C L}$ to denote the link cost of corresponding broadcast link and cooperative link, $\eta_{S, j}$ to denote the required energy for $N_{S}$ to transmit a packet to $\mathbf{C S}_{j, k}$, and $P_{j, i}, \eta_{j, i}$ to denote the required power and required energy per packet of the $i$-th relay in $\mathbf{C S}_{j, k}$, respectively. Similar to (6) (10), we have

$$
L C_{B L, j}=\max _{\forall R_{i} \in \mathbf{C S}_{j, k}} L C_{S, i}
$$

$$
\begin{gathered}
P_{j, i}=\left(\frac{1}{L C_{i, D}}\right) /\left(\sum_{\forall R_{i} \in \mathbf{C S}_{j, k}} \frac{1}{L C_{i, D}}\right)^{2} \\
L C_{j, C L}=\sum_{\forall R_{i} \in \mathbf{C S}_{j, k}} P_{j, i}=1 /\left(\sum_{\forall R_{i} \in \mathbf{C S}_{j, k}} \frac{1}{L C_{i, D}}\right) . \\
\eta_{S, j}=L C_{B L, j} \times \frac{a}{W \times \log _{2}\left(1+S N R_{\min }\right)} \\
\eta_{j, i}=P_{j, i} \times \frac{a}{W \times \log _{2}\left(1+S N R_{\min }\right)} .
\end{gathered}
$$

We use $\varepsilon_{S}, \varepsilon_{i}$ to denote the residual energy of $N_{S}$ and Relay $R_{i}(1 \leq i \leq N)$. We use $V_{j}$ to denote the virtual number of packets that could be transmitted from $N_{S}$ to $\mathbf{C S}_{j, k}$, and then to $N_{D}$ using their current residual energy, i.e.,

$$
V_{j}=\min \left(\frac{\varepsilon_{S}}{\eta_{S}}, \min _{\forall R_{i} \in \mathbf{C S}_{j, k}} \frac{\varepsilon_{i}}{\eta_{i}}\right) .
$$

where $\eta_{S}, \eta_{i}$ are the required energy for $N_{S}$ and $R_{i}$ to transmit a packet under the current channel condition.

For a $M$-relay set $\mathbf{R S}$, there are $\left(\begin{array}{c}M \\ k\end{array}\right)$ possible subsets of size $k$. For energy efficiency, we use the following criterion in choosing which subset for cooperative transmission,

$$
i=\arg _{1 \leq j \leq\left(\begin{array}{c}
M \\
k
\end{array}\right)} \max V_{j} .
$$

The above metric tells that, given the residual energy, the cooperative set $\mathbf{C S}_{i, k}$ which maximizes the number of (virtually) transmittable packets will be selected. According to (16) and (17), when a relay has relatively low residual energy, the cooperative sets that contain this relay will have relatively low, (virtually) transmittable packet numbers, which would make them less likely to be chosen for cooperative transmission. By this, the REACT algorithm avoids overly-used relays while it still benefits from the traditional cooperative transmission technique, and thus improves efficiency. Obviously, REACT incorporates both opportunistic and cooperative transmission 
techniques, making it outperform the the traditional cooperative transmission method.

Since $a /\left(W \times \log _{2}\left(1+S N R_{\min }\right)\right)$ is constant, the metric defined in (17) can be further expressed as

$$
i=\arg _{1 \leq j \leq\left(\begin{array}{c}
M \\
k
\end{array}\right)} \max \left\{\min \left(\frac{\varepsilon_{S}}{L C_{B L, j}}, \min _{\forall R_{i} \in \mathbf{C S}_{j, k}} \frac{\varepsilon_{i}}{P_{j, i}}\right)\right\} .
$$

(18) is used in the REACT algorithm to determine which cooperative subset should be chosen for cooperative transmission. Algorithm 1 is the pseudo-code of the proposed REACT algorithm.

In Algorithm 1, the initial energy of $N_{S}, R_{i}$ are denoted as $E_{S}, E_{i}$. Considering that in practice, not all energy of a node/relay can be exclusively used for data transmission, for example, some fraction of energy must dedicate to ranging, carrier sensing and/or signaling, etc., we introduce energy threshold $\delta$ in the algorithm to indicate that when the residual energy of a node/relay falls below $\delta$, the node/relay is considered dead and will not participate in the REACT algorithm. When a relay is dead, it will be removed out of the relay set RS, and the algorithm will then re-calculate all the $\left(\begin{array}{c}M \\ k\end{array}\right)$ possible cooperative subsets for the updated relay set RS. When all relays are dead, $N_{S}$ will directly transmit to $N_{D}$. When the source node $N_{S}$ is dead, the algorithm will then terminate. We would like to point out that the input parameter $r$ in the REACT algorithm is the number of packets that can be sent during a slot, i.e., $r=T /(2 a) \times W \times \log _{2}\left(1+S N R_{\text {min }}\right)$ for relay-aided transmission $\left(N_{S} \rightarrow C S_{j, k} \rightarrow N_{D}\right)$, and $r=T / a \times W \times \log _{2}\left(1+S N R_{\text {min }}\right)$ for direct transmission $\left(N_{S} \rightarrow N_{D}\right)$

We now conduct simulations to evaluate the REACT algorithm.

\section{Simulations}

In the simulations, the cooperative transmission method in [8] is used as the baseline model for comparison. The channel gain in wireless environment depends on the path loss factor, the fast fading, and the slow fading (log-normal shadow fading). In most cases, these three phenomena are assumed to be independent. Fast fading is caused by multi-path propagation, while slow fading or shadow fading, is caused by obstacles in the propagation path between two endpoints of a link. For relay-aided transmission shown in Fig. 1 or Fig. 2, line-of-sight (LOS) communication between is typically assumed for both source to relay and relay to destination transmission. Hence, we do not need to consider the lognormal shadow fading here. In fact, the REACT algorithm presented here does not care whether there is fading or not.

We use the topology in Fig. 3 for our simulation. There are 8 relays located between $N_{S}$ and $N_{D}$. Nodes and relays are placed in an area of $80 \times 120 \mathrm{~m}^{2}$. The simulation setup is as follows:

- Initial energy, $E_{S}=E_{i}=10 \mathrm{~J}$.

- Minimum energy threshold, $\delta_{S}=\delta_{i}=1 \mathrm{~J}$.

- Bandwidth $W=3.5 \mathrm{MHz}$.

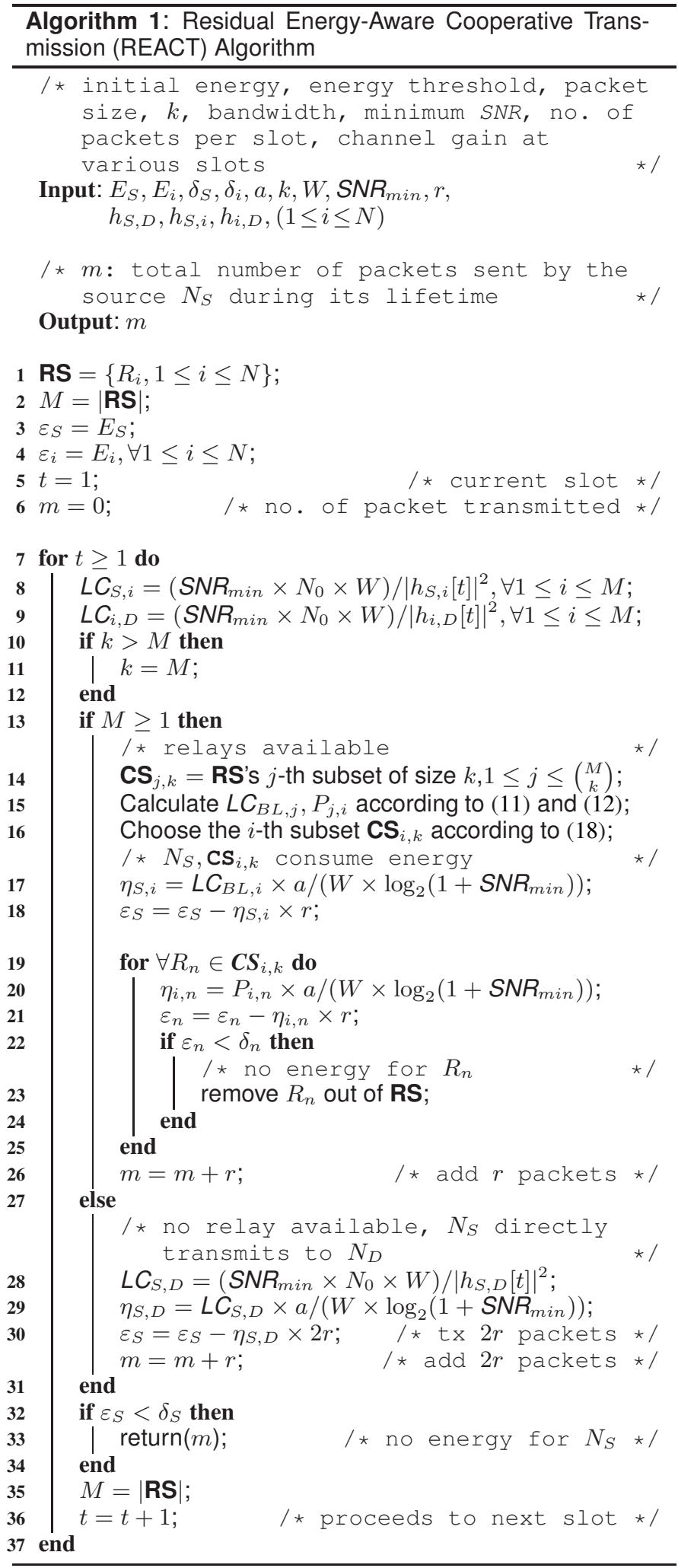

- $S N R_{\min }=2.3 \mathrm{~dB}$. This corresponds to a rate of $5 \mathrm{Mbps}$.

- Packet size $a=8000$ bits.

- Path loss exponent $\alpha=3.0$.

- Slot duration $T=3.2 \mathrm{~ms}$. 


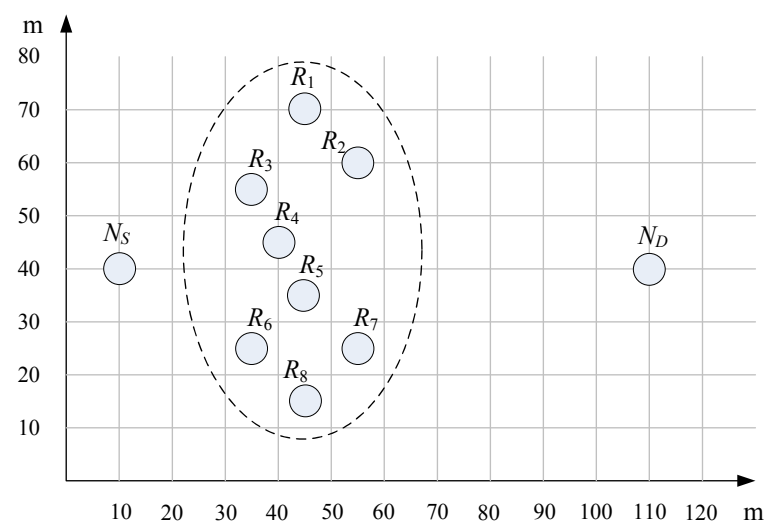

Fig. 3. Relay-Aided Cooperative Transmission in Wireless Networks

We consider the following two simulation scenarios.

\section{A. Path Loss plus Rayleigh Fading}

In this scenario, the path loss factor and the fast rayleigh fading contribute to the channel gain, i.e.,

$$
h_{i, j}=\sqrt{P L_{d_{0}} \times\left(\frac{d_{0}}{d_{i, j}}\right)^{\alpha}} \times g_{i, j} .
$$

where $g_{i, j}$ is the fast Rayleigh fading characterized by a zeromean unit-variance complex Gaussian random variable and assumed to be independent for different nodes/relays, $P L_{d_{0}} \times$ $\left(d_{0} / d_{i, j}\right)^{\alpha}$ denotes the propagation loss of the transmission power, $d_{i, j}$ is the LOS distance from Node/Relay $i$ to $j, \alpha$ is the path loss exponent (typically $2 \sim 5$ ), $d_{0}$ is the reference distance, $P L_{d_{0}}$ is the reference propagation loss at $d_{0}$.

Fig. 4 illustrated the high efficiency of REACT. In Fig. 4, we plot the number of packets transmitted by $N_{S}$ during its lifetime for various size of cooperative set. The REACT algorithm is the purely opportunistic transmission method (i.e., no cooperative transmission) for CS size $k=1$, and is the traditional cooperative transmission method (i.e., all relays participate in the cooperative transmission) for $k=8$. The green-line is the simulation result from the REACT algorithm while the red-line is the one from the baseline model. We can see that the improvement is significant. For example, the REACT algorithm produces about 11 times the amount of packets transmitted by $N_{S}$ during its lifetime when the size of $\mathbf{C S}$ is 2, and about 7 times when $k=4$ compared to the existing method.

It is known that the benefit of opportunistic resource allocation comes from channel fluctuation (e.g., fading). Similarly, the high efficiency of REACT is (partially) from fading as REACT opportunistically chooses a cooperative subset. We now evaluate the REACT algorithm when there is no fading.

\section{B. Only Path Loss, No Fading}

In this scenario, only path loss contributes to the channel gain, i.e., $h_{i, j}=\sqrt{P L_{d_{0}} \times\left(d_{0} / d_{i, j}\right)^{\alpha}}$. We use the same topology shown in Fig. 3.

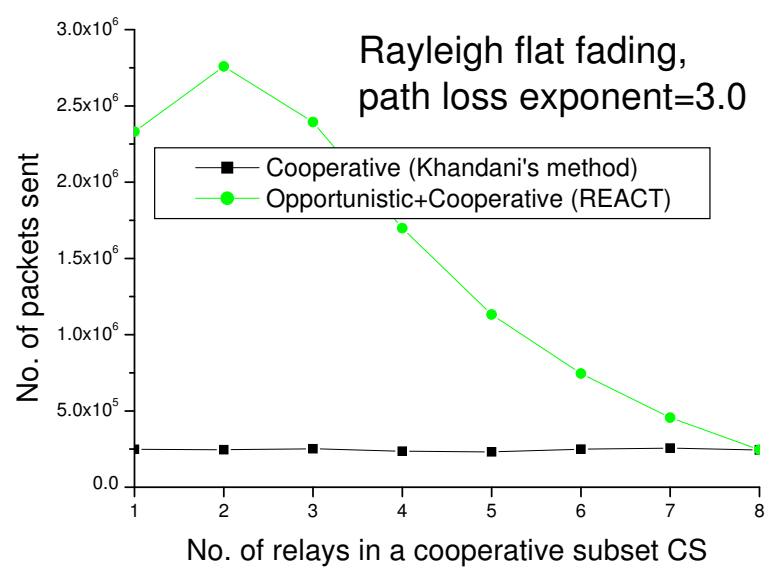

Fig. 4. Total number of packets transmitted by $N_{S}$ : REACT vs baseline method (fading case)

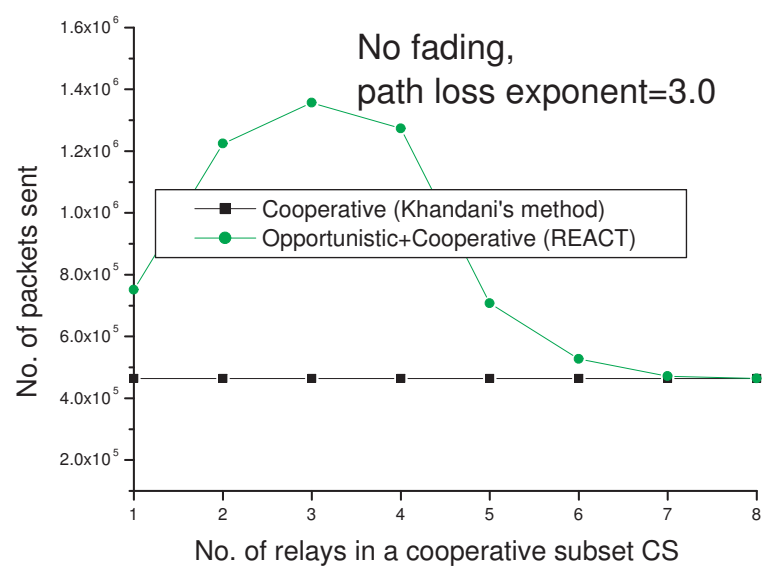

Fig. 5. Total number of packets transmitted by $N_{S}$ : REACT vs baseline method (no fading)

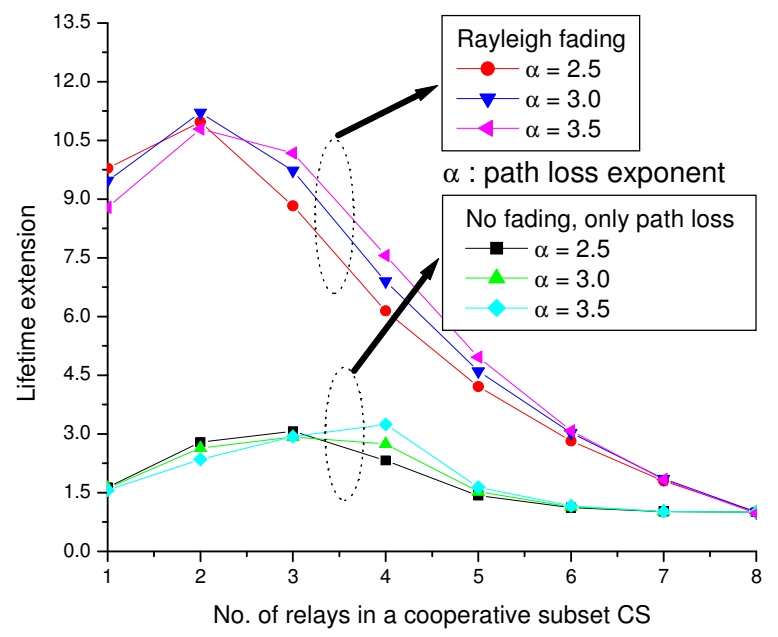

Fig. 6. Lifetime extension of REACT

Similar to the fading case, we depict in Fig. 5 the simulation results comparing our algorithm and the traditional one. Note that since all relays are involved in the transmission at each slot and there is no fading in this scenario, the simulation 
results for Khandani's method will keep the same for all simulation runs. From Fig. 5 we can see that the performance improvement of the REACT algorithm is still remarkable in this case. In fact, the difference of the residual energy among nodes makes the selection of CS "opportunistic". In this sense, REACT artificially introduces "channel fluctuation" and thus improves performance even when there is no fading.

We also plot in Fig. 6 the lifetime extension of REACT for various path loss exponent, where the lifetime extension is defined as the ratio of the lifetime of $N_{S}$ under the REACT algorithm to the one under the traditional method. We can see the REACT algorithm produces a lifetime extension of about $2.3 \sim 7.6$ when the size of the cooperative subset is $\frac{1}{2}$ of the total number of relays. Fig. 6 also showed that one or two relays are typically enough for REACT to perform well in fading scenarios

In addition, we would like to point out that, in REACT, the benefit of cooperative transmission increases with $k$. On the other hand, the benefit of opportunistic transmission in REACT decreases with $k$ when $k$ is above some point. This trade-off between the cooperative transmission and opportunistic transmission is also seen in Fig. 4 , Fig. 5 and Fig. 6.

\section{CONCLUSION}

In this paper, we formulated the problem of maximizing the lifetime of a battery-operated node as maximizing the amount of packets transmitted by the node. We proposed a relay selection algorithm to choose a subset of relays in the relay set taking consideration of the residual power of each node. In our method, we avoid the selection of overly-used nodes in each relay transmission stage, and thus is more energy-efficient. We followed the method proposed in [8] for the power allocation inside a cooperative subset and used it as a baseline model for comparison. Simulation results reveal much improvement in lifetime extensions with our REACT algorithm.

\section{REFERENCES}

[1] B. Seshasayee and K. Schwan, "Energy-effcient device scheduling through contextual timeouts," Georgia Institute of Technology, Tech. Rep., 2005.

[2] W. Ye, J. Heidemann, and D. Estrin, "An energy-efficient mac protocol for wireless sensor networks," in Proc. INFOCOM 2002. The 21st Joint Conference of the IEEE Computer and Communications Societies. IEEE, vol. 3, 2002, pp. 1567-1576 vol.3.

[3] J.-H. Chang and L. Tassiulas, "Energy conserving routing in wireless adhoc networks," in Proc. INFOCOM 2000. The 9th Joint Conference of the IEEE Computer and Communications Societies. IEEE, vol. 1, 2000, pp. 22-31 vol.1.

[4] J. E. Wieselthier, G. D. Nguyen, and A. Ephremides, "Algorithms for energy-efficient multicasting in static ad hoc wireless networks," $A C M$ Mobile Networks and Applications (MONET), vol. 6, no. 3, pp. 251-263, 2001.

[5] J. N. Laneman, D. N. C. Tse, and G. W. Wornell, "Cooperative diversity in wireless networks: Efficient protocols and outage behavior," IEEE Transactions on Information Theory, vol. 50, no. 12, pp. 3062-3080, 2004.

[6] A. Sendonaris, E. Erkip, and B. Aazhang, "User cooperation diversity. part i. system description," IEEE Transactions on Communications, vol. 51, no. 11, pp. 1927-1938, November 2003.

[7] A. Bletsas, A. Khisti, D. P. Reed, and A. Lippman, "A simple cooperative diversity method based on network path selection," IEEE Journal on Selected Areas in Communications, vol. 24, no. 3, pp. 659-672, March 2006.

[8] A. E. Khandani, J. Abounadi, E. Modiano, and L. Zheng, "Cooperative routing in static wireless networks," IEEE Transactions on Communications, vol. 55, no. 11, pp. 2185-2192, November 2007.

[9] Y.-S. Tu and G. J. Pottie, "Coherent cooperative transmission from multiple adjacent antennas to a distant stationary antenna through awgn channels," in Proc. IEEE 55th Vehicular Technology Conference VTC Spring 2002, vol. 1, 2002, pp. 130-134 vol.1. 